\title{
Adaptive Range-Based Power Control for Collision Avoidance in Wireless Ad Hoc Networks
}

\author{
Kuei-Ping Shih, Yen-Da Chen, and Chau-Chieh Chang \\ Department of Computer Science and Information Engineering, Tamkang University, Tamshui 251, Taipei, Taiwan \\ Email: kpshih@mail.tku.edu.tw
}

\begin{abstract}
The paper analyzes the relationships among the transmission range, carrier sensing range, and interference range in case that power control is adopted and proposes an adaptive range-based power control (ARPC) MAC protocol for wireless ad hoc networks to avoid collisions. Based on the analysis results, four mechanisms, STRC, RTRC, SCRC, and RCRC are proposed to prevent from collisions. The paper further analyzes the superiority of each mechanism under certain situations and proposes the ARPC MAC protocol to make use of the advantages of the four mechanisms to avoid collisions. The proposed protocol can not only reduce energy consumption of STAs, but also prevent from collisions. Simulation results also verify the advantages of the proposed protocol.
\end{abstract}

\section{INTRODUCTION}

A wireless ad hoc network is a network temporarily formed by a collection of stations (STAs) without relying on any established infrastructure. Efficient energy management is much critical for wireless ad hoc networks because STAs are usually battery-powered. In general, power control mechanism can decrease STAs energy consumption, and further increase the network lifetime. Therefore, a lot of researchers pay their attention on power control recently [1]-[10].

In [1], [2], similar power control mechanisms are proposed, where RTS/CTS are sent at the maximum power level $\left(P_{\max }\right)$, and DATA/ACK are sent at the minimum necessary power level $\left(P_{\text {min }}\right)$ as long as the receiver's received signal to noise ratio (SNR) is above a threshold value. These mechanisms are referred to the basic power control mechanism. Unfortunately, when the basic power control mechanism is used, two kinds of collision problems may happen [3], [6].

A STA may interfere with current transmission due to the shrink of the carrier sensing range if power control is adopted. This collision is termed as the first collision problem [3]. FEC code and additional transceiver for busy tone transmission are required to prevent from the first collision problem in [4], [5], respectively. In [3], the schemes similar to the basic power control mechanism are proposed, which periodically uses $P_{\max }$, instead of $P_{\min }$, for DATA transmission to avoid the first collision problem. The scheme in [3] needs no additional hardware cost. However, this protocol may not prevent from the second collision problem, which is also the most important problem to be solved.

A STA interfering with current transmission due to the expansion of the interference range if power control is adopted causes the second collision problem. The collision problem is also termed the POINT problem [6]. In [6]-[10], the POINT problem can be solved completely. In [6], [8]-[10], CTS is exploited to avoid the POINT problem. In [8], [9], a power level larger than $P_{\max }$ is required for CTS transmission. Thus, the mechanisms in [8], [9] can tolerate of larger interference, and DATA can be transmitted in a lower power level. On the other hand, in [7], a special topology, mesh network, is assumed.

In the paper, four mechanisms, Sender's Transmission Range Cover (STRC), Receiver's Transmission Range Cover (RTRC), Sender's Carrier-sensing Range Cover (SCRC), and Receiver's Carrier-sensing Range Cover (RCRC) are proposed. These four mechanisms have their superiorities in different scenarios. Hence, an adaptive range-based power control (ARPC) MAC protocol combining the advantages of RTRC, SCRC, and RCRC is proposed as well. Simulation results show that ARPC achieves better network throughput and lower packet loss rate than the related work. ARPC can indeed avoid the POINT problem and enhance the network throughput of wireless ad hoc networks.

The rest of this paper is organized as follows. Section II formally derives the transmission range, carrier sensing range, and the interference range induced by some power. STRC, RTRC, SCRC, and RCRC schemes are formulated and analyzed in Section III. In addition, the energy consumptions of STRC, RTRC, SCRC, and RCRC schemes are compared in this section. In Section IV, an adaptive range-based power control (ARPC) MAC protocol is proposed. Simulation results are presented in Section V. Section VI concludes the paper.

\section{PRELIMINARIES}

The definitions of the transmission range, the carrier sensing range, and the interference range have been defined in previous work [11]. For completeness, the definitions of these three ranges are restated as follows.

Definition 1 (Transmission Range, $\boldsymbol{T R}$ ): is defined as the range within which a packet can be successfully received and correctly identified.

Definition 2 (Carrier-sensing Range, CR): is defined as the range within which the signal can be detected, and the medium will be set in busy state.

Definition 3 (Interference Range, IR): is defined as the range within which the receiving STA will be interfered by other STAs and thus suffer a packet loss. 
$T R$ and $C R$ much depend on the antenna sensitivity. In general, the antenna sensitivity of $T R$ is usually higher than or equal to the antenna sensitivity of $C R$. Without loss of generality, we assume $C R=\delta^{*} T R$, where $\delta \geq 1$. In addition, $T R, C R$, and $I R$ also vary according to the transmission power of the sender. In this paper, $T R(P), C R(P)$ and $I R(P)$ denote $T R, C R$, and $I R$ induced by the transmission power $P$ of the sender, respectively. $T R(P)$ and $I R(P)$ have been derived in [6]. We conclude these derivations as the following theorem.

Theorem 1: Suppose the radio propagation model is twoway ground model [12]. The distance between the sender, $S$, and the receiver, $R$, is denoted as $D_{S R}$. $S$ uses the power $P_{S}$ to transmit. Therefore, the transmission range of $S, T R\left(P_{S}\right)$, the carrier-sensing range of $S, C R\left(P_{S}\right)$, and the interference range of $R, I R\left(P_{S}\right)$, are as follows.

$$
\begin{aligned}
T R\left(P_{S}\right) & =\left(c \frac{P_{S}}{P_{\alpha}}\right)^{\frac{1}{4}}, \\
C R\left(P_{S}\right) & =\delta * T R\left(P_{S}\right)=\delta *\left(c \frac{P_{S}}{P_{\alpha}}\right)^{\frac{1}{4}}, \\
I R\left(P_{S}\right) & =\left(\frac{c^{\prime} P_{\text {max }}}{\frac{c P_{S}}{D_{S R}^{4} S N R_{\text {thold }}}-P_{C N}}\right)^{\frac{1}{4}},
\end{aligned}
$$

where $c$ and $c^{\prime}$ are two constants, $P_{\alpha}=\max \left\{P_{\text {thold }}, P_{N} *\right.$ $\left.S N R_{\text {thold }}\right\}, P_{N}$ is the maximum tolerant noise, $P_{C N}$ is the current noise, $P_{\text {thold }}$ and $S N R_{\text {thold }}$ are respectively the received signal strength threshold and the SNR threshold such that a STA can successfully receive and identify a packet, and $P_{\max }$ is the maximum transmission power.

For simplicity, let the current noise, $P_{C N}$, and constants, $c$ and $c^{\prime}$, be ignored. In practice, when $P_{C N}=0, P_{N} * S N R_{\text {thold }}$ is always smaller than $P_{\text {thold }}$. Thus, we can get $P_{\alpha}=P_{\text {thold }}$. In addition, $S N R_{\text {thold }}$ and $\delta$ are set to 10 and 2 [11], respectively. As a result, the following corollary is obtained accordingly.

Corollary 1: Suppose $S N R_{\text {thold }}$ and $\delta$ are set to 10 and 2, respectively. If the current noise, $P_{C N}$, constant $c$ and constant $c^{\prime}$ are ignored, Theorem 1 can be simplified as follows.

$$
\begin{aligned}
& \operatorname{TR}\left(P_{S}\right)=\left(\frac{P_{S}}{P_{\text {thold }}}\right)^{\frac{1}{4}}, \\
& C R\left(P_{S}\right)=2 * T R\left(P_{S}\right)=2 *\left(\frac{P_{S}}{P_{\text {thold }}}\right)^{\frac{1}{4}}, \\
& I R\left(P_{S}\right)=1.78 *\left(\frac{P_{\text {max }}}{P_{S}}\right)^{\frac{1}{4}} D_{S R} .
\end{aligned}
$$

According to Theorem 1, the $I R$ of the receiver is deeply related to the transmission power of the sender. When $P_{\min }$ is adopted, the $I R$ of the receiver will expand. Therefore, the POINT problem may happen [6]. For completeness, the definition of the POINT problem is described as follows.

Definition 4 (POINT Problem): In power control mechanism, $S$ and $R$ use $P_{\max }$ to exchange RTS/CTS and use $P_{S}$ to exchange DATA/ACK, where $P_{S} \leq P_{\max }$. Suppose $D_{S R} \leq 0.56 * T R\left(P_{\max }\right)$. In this case, $\operatorname{IR}\left(P_{S}\right) \leq T R\left(P_{\max }\right)$

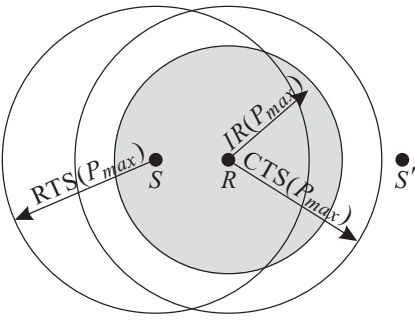

(a)

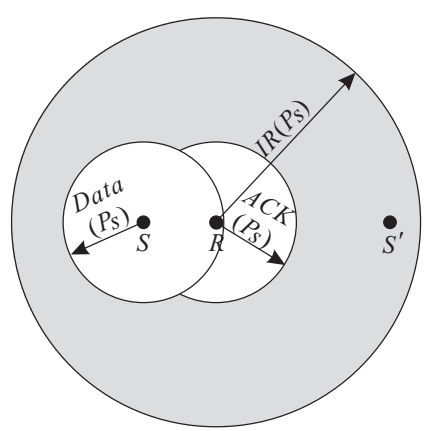

(b)
Fig. 1. The POINT problem. (a) $D_{S R} \leq 0.56 T R\left(P_{\max }\right) . S$ and $R$ use $P_{\max }$ to exchange RTS/CTS. The gray area is $I R\left(P_{\max }\right)$, which is smaller than $T R\left(P_{\max }\right)$ since $D_{S R} \leq 0.56 T R\left(P_{\max }\right)$. $S^{\prime}$, a source of interference, is outside both $T R\left(P_{\max }\right)$ and $I R\left(P_{\max }\right)$. (b) $S$ and $R$ use the reduced power, $P_{S}$, to exchange Data/ACK. $I R\left(P_{S}\right)$ will be larger than $T R\left(P_{\max }\right)$ due to the reduction of the sender's power strength. As a result, $S^{\prime}$ is within $\operatorname{IR}\left(P_{S}\right)$ and may cause collision.

[11]. Assume $S^{\prime}$ is a STA out of the $T R\left(P_{\max }\right)$ of $R$. In other words, $S^{\prime}$ can not overhear CTS transmitted by $R$. In the meanwhile, $S^{\prime}$ intends to transmit using $P_{\max }$. If $S$ uses $P_{S}$ to transmit to $R, I R$ of $S$ will expand and $S^{\prime}$ will be within $\operatorname{IR}\left(P_{S}\right)$. Therefore, $S^{\prime}$ will interfere $R$. The collision is denoted as $P O$ wer control $I N$ duced hidden Terminal problem (POINT problem).

\section{RANGe Cover Mechanisms}

In this section, four techniques, SCRC, RCRC, STRC, and RTRC, are proposed to avoid the POINT problem. In addition, the energy consumption of these four schemes is also analyzed in this section.

\section{A. Sender's Carrier-sensing Range Cover Mechanism (SCRC)}

1) Concept of SCRC: A physical carrier-sense mechanism is provided to avoid collisions in IEEE 802.11 standard. The STAs within $C R$ of the sender can detect the signal and set the medium in busy state to defer their transmissions. Thus, $C R$ of the sender can be used to solve the POINT problem if $I R\left(P_{S C R C}\right)$ of the receiver can be completely covered by $C R\left(P_{S C R C}\right)$ of the sender where $P_{S C R C}$ is the power level used by the sender to transmit. Therefore, we have

$$
C R\left(P_{S C R C}\right) \geq D_{S R}+I R\left(P_{S C R C}\right) .
$$

\section{2) Derivation of Transmission Power and Restriction:}

- Derivation of Transmission Power

Based on the previous description, the POINT problem can be avoided if the sender adopts the power level, $P_{S C R C}$, to transmit DATA. According to Corollary 1 and Eq. (7), $P_{S C R C}$ can be obtained as follows.

$$
P_{S C R C} \geq\left(\frac{1}{4} P_{\text {thold }}^{\frac{1}{4}}\left(D_{S R}+\left(\beta D_{S R}\right)^{\frac{1}{2}}\right)\right)^{4},
$$

where $\beta=D_{S R}+14.24 * P_{\text {thold }}^{\frac{-1}{4}} P_{\text {max }}^{\frac{1}{4}}$. As a result, the sender can use $P_{S C R C}$ to transmit DATA to avoid the POINT problem completely. 

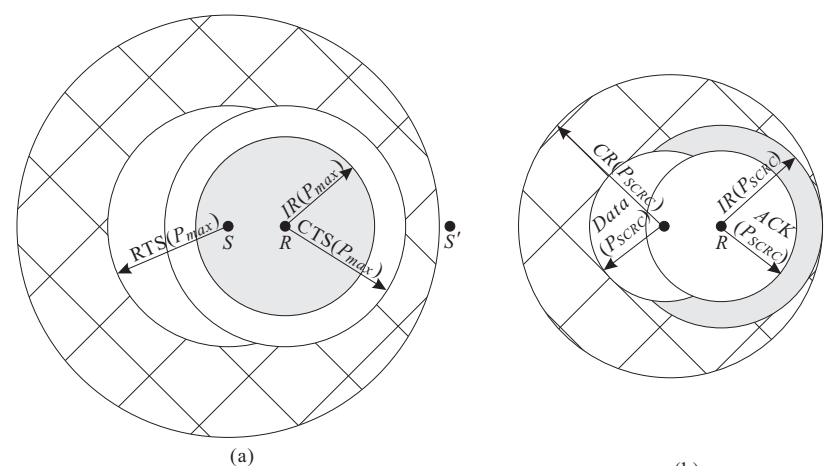

(b)

Fig. 2. The concept of SCRC. (a) $S$ and $R$ use $P_{\max }$ to exchange RTS/CTS. The gray area is $I R\left(P_{\max }\right)$, which, in this case, is covered by $C R\left(P_{\max }\right)$ since $D_{S R} \leq 0.72 T R\left(P_{\max }\right)$. $S^{\prime}$, a source of interference, is outside both $T R\left(P_{\max }\right)$ and $I R\left(P_{\max }\right)$. (b) $S$ and $R$ use $P_{S C R C}$ to exchange DATA/ACK, where $P_{S C R C}$ is set to the power level that satisfies $C R\left(P_{S C R C}\right)=D_{S R}+I R\left(P_{S C R C}\right)$.

- Restriction

Since $P_{S C R C}$ is limited to $P_{\max }$, if $P_{S C R C}=P_{\max }$, we can get

$$
D_{S R} \leq 0.72 * T R\left(P_{\max }\right) .
$$

That implies when the $D_{S R}$ is smaller than $0.72 *$ $T R\left(P_{\max }\right)$, the $C R$ of DATA can fully cover the $I R$ of the receiver.

3) SCRC MAC Protocol: The concept of SCRC MAC protocol is shown in Fig. 2. Suppose STA $S$ wants to communicate with $R$. Following CSMA/CA mechanism, $S$ successfully contends the medium access right and uses $P_{\max }$ to send RTS to $R$. If $R$ successfully receives the RTS packet, by Eq. (8), $R$ evaluates $P_{S C R C}$ and attaches this information in its CTS packet. $R$ then uses $P_{\max }$ to reply CTS to $S$. Finally, $S$ and $R$ use $P_{S C R C}$ to exchange DATA and ACK. Therefore, SCRC can totally avoid the POINT problem.

\section{B. Receiver's Carrier-sensing Range Cover Mechanism $(R C R C)$}

1) Concept of RCRC: The main idea of RCRC is to let $C R$ of CTS cover $I R$ of the receiver to avoid the POINT problem. That is, it requires

$$
I R \leq C R
$$

According to Eq. (6), IR is greatly related to the sender's transmission power. Let $P_{\min }$ be the minimum power of the sender required transmitting to the receiver. If the transmission power is $P_{\text {min }}$, it will cause the maximum $I R$. The following theorem derives the maximum $I R$ caused by $P_{\text {min }}$

Theorem 2: When the sender adopts $P_{\min }$ to transmit, $I R$ of the receiver, $I R\left(P_{\min }\right)$, will be always equal to $1.78 * T R\left(P_{\max }\right)$, which is independent of $D_{S R}$.

Proof: Since $P_{\min }$ is the minimum necessary transmission power level for the sender to transmit, therefore, $T R\left(P_{\min }\right)=D_{S R}$. According to Eq. (4) in Corollary 1, $P_{\min }$ can be obtained as below.

$$
P_{\text {min }}=P_{\text {thold }} \cdot D_{S R}^{4} \text {. }
$$

Substituting $P_{\min }$ by the above equation into Eq. (6), $I R\left(P_{\min }\right)$ of the receiver can be obtained as follows.

$$
I R\left(P_{\text {min }}\right)=1.78\left(\frac{P_{\text {max }}}{P_{\text {thold }}}\right)^{\frac{1}{4}} .
$$

It implies

$$
\operatorname{IR}\left(P_{\min }\right)=1.78 * T R\left(P_{\max }\right) .
$$

Obviously, $D_{S R}$ does not affect the interference range if $S$ uses $P_{\min }$ to transmit to $R$.

Therefore, if $C R$ of CTS can cover the maximum $I R$, the POINT problem can be avoided. According to Corollary $1, C R\left(P_{\max }\right)$ is twice of $T R\left(P_{\max }\right)$. However, based on Eq. (13), the maximum $I R$ is 1.78 times $T R\left(P_{\max }\right)$. Hence, $I R\left(P_{\min }\right)<C R\left(P_{\max }\right)$. As a result, $I R\left(P_{\min }\right)$ of the receiver is always smaller than $C R\left(P_{\max }\right)$ of CTS. That implies the receiver needs not transmit CTS in $P_{\max }$. In other words, the receiver can use a smaller power, say $P_{R C R C}$, to transmit CTS instead of $P_{\max }$. However, $P_{R C R C}$ should satisfy

$$
I R\left(P_{\min }\right) \leq C R\left(P_{R C R C}\right) .
$$

As a result, the receiver can use $P_{R C R C}$ to reply CTS and the sender can use $P_{\min }$ to transmit DATA to avoid the POINT problem.

2) Derivation of Transmission Power and Restriction:

- Derivation of Transmission Power According to Corollary 1 and Eq. (14), we can get

$$
1.78 *\left(\frac{P_{\max }}{P_{\min }}\right)^{\frac{1}{4}} D_{S R} \leq 2 *\left(\frac{P_{R C R C}}{P_{\text {thold }}}\right)^{\frac{1}{4}} .
$$

Thus, $P_{R C R C}$ can be obtained as follows.

$$
P_{R C R C} \geq \frac{0.89^{4} * P_{\max } * D_{S R}^{4} * P_{\text {thold }}}{P_{\min }} .
$$

The $P_{R C R C}$ derived from Eq. (15) can ensure that $I R\left(P_{\text {min }}\right) \leq C R\left(P_{R C R C}\right)$. However, $P_{R C R C}$ still needs to make sure that CTS of $R$ can be received by $S$. Therefore, $P_{R C R C}$ still has to satisfy $T R\left(P_{R C R C}\right) \geq D_{S R}$. Thus, by Eq. (4), $P_{R C R C} \geq D_{S R}^{4} * P_{\text {thold }}$. Consequently,

$$
P_{R C R C} \geq \max \left(\frac{0.89^{4} * P_{\max }}{P_{\min }}, 1\right) * D_{S R}^{4} * P_{\text {thold }} .
$$

- Restriction

Since $I R\left(P_{\min }\right)<C R\left(P_{\max }\right), P_{R C R C}$ is always smaller than $P_{\max }$. Dislike SCRC, RCRC has no power restriction. However, RCRC has a restriction on DATA length. In IEEE 802.11 standard, a STA which does not correctly receive the last frame shall defer a period of time, EIFS, after the medium is determined to be idle. Since, in RCRC, $I R$ is covered by $C R$ of CTS, the interfering STAs located in $C R$ of the receiver will set an EIFS to defer its transmission. Therefore, in RCRC, the duration of the DATA transmission should be shorter than an EIFS. Otherwise, collisions may happen. Consequently,

$$
\frac{L \times 8}{r}+\text { aSIFSTime } \leq \text { aEIFSTime, }
$$




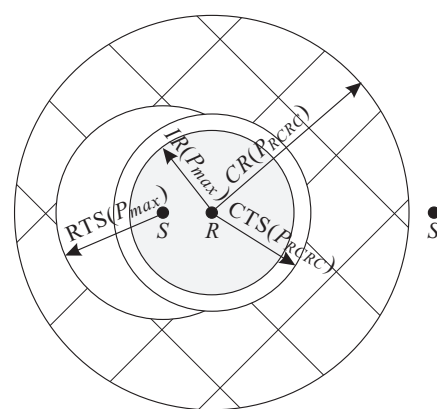

(a)

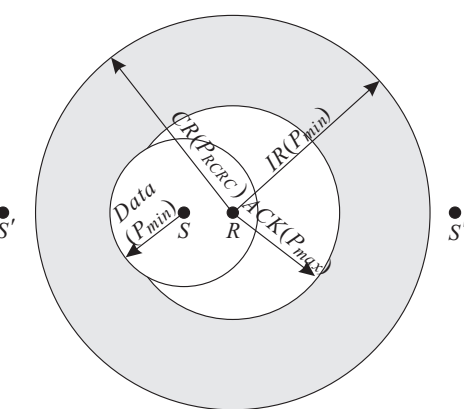

(b)
Fig. 3. The concept of RCRC. (a) $S$ uses $P_{\max }$ to send RTS and $R$ adopts $P_{R C R C}$ to reply CTS, where $P_{R C R C}$ is set to the power level such that $I R\left(P_{\min }\right)=C R\left(P_{R C R C}\right)$. $S^{\prime}$ is outside $I R\left(P_{\min }\right)$. (b) $S$ uses $P_{\min }$ to send DATA and $R$ uses $P_{\max }$ to reply ACK. $S^{\prime}$ is still outside $I R\left(P_{\min }\right)$.

where $L$ is the DATA length in bytes and $r$ is the transmission rate in Mbps. On the basis of IEEE 802.11 standard, EIFS $=$ SIFS + DIFS $+[(8 \times$ ACKsize $)$ +PreambleLength + PLCPHeaderLength] / BitRate [13], where BitRate is the PHY's lowest mandatory rate. Accordingly, EIFS is equal to $364 \mu \mathrm{s}$ under $1 \mathrm{Mbps}$ channel bit rate. In DSSS PHY, aSIFSTime is set to 10 $\mu$ s. Therefore, by Eq. (17), $L$ should satisfy that

$$
L \leq 44.25 * r .
$$

Therefore, the restriction of RCRC is that the length of DATA should be shorter than $44.25 * r$ bytes.

3) RCRC MAC Protocol: The concept of RCRC MAC protocol is shown in Fig. 3. Suppose $S$ wants to transmit to $R$. If $S$ successfully contends the medium access right and the DATA length to be sent is shorter than or equal to $44.25 * r$ bytes, $S$ uses $P_{\max }$ to send RTS. If $R$ successfully receives the RTS packet, by Eq. (16), $R$ evaluates $P_{R C R C}$ and uses $P_{R C R C}$ to reply CTS to $S$. As a result, $S$ and $R$ use $P_{\text {min }}$ and $P_{\max }$ to exchange DATA and ACK packets, respectively. In such a way, not only the sender can adopt the minimum power to transmit DATA and save its power consumption, but also the POINT problem can be completely and effectively avoided.

C. Sender's Transmission Range Cover Mechanism (STRC) and Receiver's Transmission Range Cover Mechanism (RTRC)

In IEEE 802.11 standard [13], the virtual carrier-sense mechanism is provided to avoid hidden terminal problem. Under virtual carrier-sense mechanism, the hidden STAs can predict the subsequent traffic on the medium based on the duration information announced in RTS or CTS to defer their transmissions. Therefore, STRC or RTRC figure out an appropriate power level, $P_{S T R C}$ or $P_{R T R C}$, for the sender to transmit DATA, such that $I R\left(P_{S T R C}\right)$ or $I R\left(P_{R T R C}\right)$ can be covered by the $T R$ of RTS or the $T R$ of CTS, respectively. However, due to the lack of space, the paper just shows the derivation results of $P_{S T R C}$ and $P_{R T R C}$ as follows.

$$
P_{S T R C} \geq 10 *\left(P_{\text {thold }}^{\frac{-1}{4}} D_{S R}^{-1}-P_{\max }^{\frac{-1}{4}}\right)^{-4}
$$

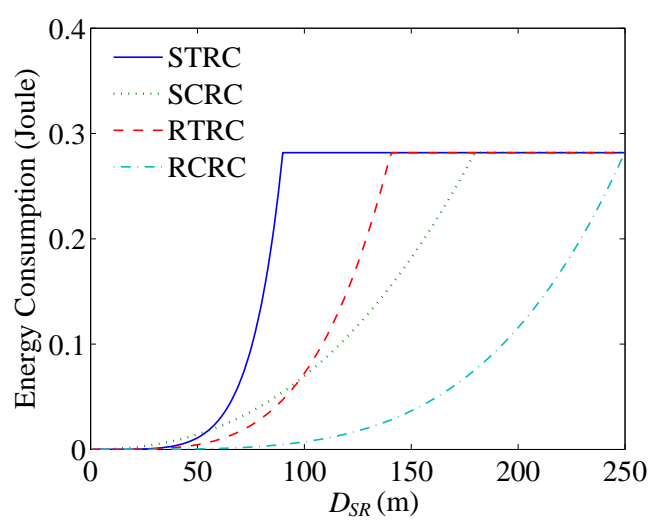

Fig. 4. Comparisons of energy consumption among STRC, RTRC, SCRC and RCRC in terms of $D_{S R}$.

and

$$
P_{R T R C} \geq 10 * D_{S R}^{4} * P_{\text {thold }}
$$

\section{Comparisons of the Four MAC Protocols}

In the previous section, $P_{S T R C}, P_{R T R C}, P_{S C R C}$, and $P_{\min }$ are adopted by STRC, RTRC, SCRC, and RCRC for the sender to send DATA, respectively. Although the energy consumption of RCRC is the least, the usage of RCRC is restricted in DATA length. Actually, the four mechanisms have their superiority at different aspects. Therefore, it is important to compare the energy consumption of the four mechanisms.

On the basis of the analysis in the previous section, the values of $P_{S T R C}, P_{R T R C}$ and $P_{S C R C}$ in terms of $D_{S R}$ are shown in Fig. 4. Obviously, the energy consumption of $P_{S T R C}$ is always higher than that of $P_{R T R C}$. It is because STRC needs more power than RTRC to cover the $I R$ of the receiver. Thus, we can conclude that RTRC is more energy efficient than STRC. As Fig. 4 indicates, the energy consumption of $P_{R T R C}$ is not always higher than that of $P_{S C R C}$. In order to get the intersection point of $P_{R T R C}$ and $P_{S C R C}$, we assume

$$
P_{R T R C}=P_{S C R C} .
$$

Take Eq. (20) and Eq. (8) into Eq. (21), the intersection point is occurred when

$$
D_{S R}=0.39 * P_{\text {thold }}^{\frac{-1}{4}} P_{\max }^{\frac{1}{4}} .
$$

From Eq. (4), Eq. (22) can be modified as follows.

$$
D_{S R}=0.39 * T R\left(P_{\max }\right) .
$$

Eq. (23) means that when $D_{S R}$ is $0.39 * T R\left(P_{\max }\right)$, the energy consumption of RTRC is the same as SCRC. Accordingly, when $D_{S R}$ is smaller than $0.39 * T R\left(P_{\max }\right)$, the energy consumption of RTRC is less than that of SCRC. On the contrary, SCRC is more energy efficient than RTRC when $D_{S R}$ is larger than $0.39 * T R\left(P_{\max }\right)$. As a consequence, the comparisons among STRC, RTRC, SCRC, and RCRC are summarized in Table I. 
TABLE I

COMPARISONS AMONG STRC, RTRC, SCRC, AND RCRC.

\begin{tabular}{c|cccl} 
& CTS & DATA & ACK & Superiority \\
\hline STRC & $P_{\max }$ & $P_{S T R C}$ & $P_{S T R C}$ & none \\
RTRC & $P_{\max }$ & $P_{R T R C}$ & $P_{R T R C}$ & $D_{S R} \leq 0.39 T R\left(P_{\max }\right)$ \\
SCRC & $P_{\max }$ & $P_{S C R C}$ & $P_{S C R C}$ & $0.39 T R\left(P_{\max }\right) \leq D_{S R}$ \\
& & & & $\leq 0.72 T R\left(P_{\max }\right)$ \\
RCRC & $P_{R C R C}$ & $P_{\min }$ & $P_{\max }$ & $L \leq 44.25 * r$ Bytes
\end{tabular}

\section{Adaptive Range-Based Power Control (ARPC) MAC PROTOCOL}

According to previous analyses, SCRC, RTRC, and RCRC have their merits in different scenarios. In this section, an adaptive range-based power control MAC protocol (ARPC) combining the advantages of the SCRC, RTRC, and RCRC is proposed for solving the POINT problem. The detailed procedure of ARPC is stated as follows.

Suppose $S$ intends to transmit to $R$. If $S$ successfully contends the medium access right, $P_{\max }$ will be adopted to send RTS. If the length of DATA is shorter than $44.25 * r$ bytes, RCRC MAC protocol is adopted for the subsequent steps of a four-way handshake, where $r$ is the transmission rate. Otherwise, $D_{S R}$ is taken into consideration for $R$ to decide which MAC protocol will be used. If $D_{S R} \geq 0.39 * T R\left(P_{\max }\right)$, SCRC will be used for the following steps of a four-way handshake. Otherwise, RTRC is adopted.

\section{Performance Evaluations}

To verify the effectiveness of SCRC, RTRC, RCRC, and ARPC protocols, the four protocols as well as IEEE 802.11 DCF [13] are simulated and compared. The metrics to be evaluated include throughput, energy consumption, and energy efficiency (throughput per joule). The simulations are conducted by $n s-2$ simulator [14]. Simulation settings are shown in Table II.

TABLE II

SiMULATION SETTINGS.

\begin{tabular}{ll}
\hline Parameter & Value \\
\hline$T R\left(P_{\max }\right)$ & $250 \mathrm{~m}$ \\
$C R\left(P_{\max }\right)$ & $500 \mathrm{~m}$ \\
Transmission rate & $1 \mathrm{Mb} / \mathrm{s}$ \\
$P_{\max }$ & $28.183 \mathrm{~mW}$ \\
$P_{\text {thold }}$ & $3.652 * 10^{-7} \mathrm{~mW}$ \\
$S N R_{\text {thold }}$ & 10 \\
Traffic model & $\mathrm{CBR}$ \\
Mean frame length & 2312 octets \\
\hline
\end{tabular}

A linear topology is employed to observe the behaviors of RTRC, SCRC, RCRC, ARPC, and IEEE 802.11 DCF, where four STAs $A, B, C$, and $D$ form a line in turn. $A, C$, and $D$ are stationary and $B$ will change its position toward $C$ in the simulation. The distances between $A$ and $D$ as well as $C$ and $D$ are respectively fixed to $800 \mathrm{~m}$ and $250 \mathrm{~m}$. The distance between $A$ and $B$ is denoted $D_{A B}$, which is varied from 10 $\mathrm{m}$ to $250 \mathrm{~m}$. There are two CBR flows, $A \rightarrow B$ and $C \rightarrow D$, in the simulation. Since the transmission probabilities of the two flows are not fair [15], the interarrival time of $A \rightarrow B$ is set to $0.00001 \mathrm{sec}$ and that of $C \rightarrow D$ is set to $0.001 \mathrm{sec}$ in the simulation.

Fig. 5(a) illustrates the energy consumption of the proposed power control protocols against IEEE 802.11 DCF. In power control mechanisms, the distance between the sender and the receiver directly affects STAs' energy consumption. The longer the distance is, the more the energy consumes. On the contrary, IEEE 802.11 DCF always consumes the maximum power, irrelevant to the distance between the sender and the receiver since no power control is adopted. By Fig. 5(a), it is obvious that the energy consumption of IEEE 802.11 DCF is the worst. On the other hand, the energy consumptions of RTRC, SCRC, and RCRC are consistent with the analyzed results shown in Fig. 4. The energy consumption of RCRC performs the best due to the minimum power for DATA transmission and a smaller power for CTS transmission. Moreover, the performance of RTRC is better than that of SCRC when $D_{A B}$ is shorter than $97.5 \mathrm{~m}$, which is equal to $0.39 * T R\left(P_{\max }\right)$. Otherwise, SCRC performs better than RTRC. Since the packet length used in the simulation is larger than $44.25 * r$, as a result, RCRC will never be adopted by ARPC. Thus, except RCRC, ARPC performs the best against RTRC, SCRC, and IEEE 802.11 DCF.

Fig. 5(b) demonstrates the throughput of the five protocols in terms of $D_{A B}$ varied from $10 \mathrm{~m}$ to $250 \mathrm{~m}$. When $D_{A B}$ is shorter than $50 \mathrm{~m}$, the two flows can transmit simultaneously without interference. Therefore, the five protocols have the same throughput. However, when $D_{A B}$ is equal to or larger than $50 \mathrm{~m}, B$ is within $C R$ of $C$. Thus, the two flows will interfere with each other. That is the reason why the throughput of the five protocols drops. Moreover, in RCRC protocol, when $D_{A B}$ is larger than $105 \mathrm{~m}, C$ is located in the $I R$ of $B$ because, in RCRC, the sender always uses the minimum power for DATA transmission and the interference range is always $1.78 * T R\left(P_{\max }\right)$, which is equal to $445 \mathrm{~m}$. As a result, when $D_{A B}$ is larger than $105 \mathrm{~m}$, the throughput of RCRC will drop again. Nevertheless, the other four protocols will not be affected by the phenomenon. However, when $D_{A B}$ is larger than $198 \mathrm{~m}, C$ is within the $I R$ of $B$ for the four protocols, except RCRC. Therefore, the throughput of the four protocols drops and the four protocols have the same throughput with that of RCRC. It is worth noting that RTRC and SCRC will use the maximum power to transmit when $D_{A B}$ is respectively larger than 0.56 and 0.72 times the maximum transmission range. As a result, RTRC, SCRC, and ARPC will have the same throughput with that of IEEE 802.11 DCF.

Fig. 5(c) compares the energy efficiencies of the five protocols in terms of $D_{A B}$ varied from $10 \mathrm{~m}$ to $250 \mathrm{~m}$. Energy efficiency is defined as the number of bits transmitted per unit of time and per unit of energy consumption, which can be obtained by dividing throughput by energy consumption. 


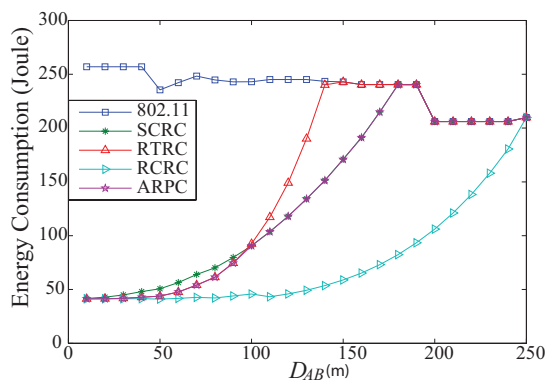

(a)

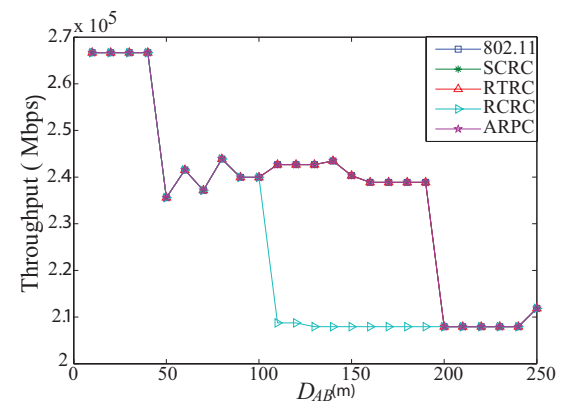

(b)

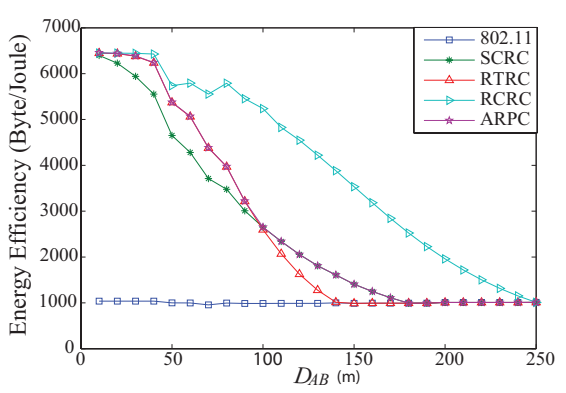

(c)

Fig. 5. RTRC, SCRC, RCRC, ARPC, and IEEE 802.11 DCF are compared for $D_{A B}$ varied from $10 \mathrm{~m}$ to $250 \mathrm{~m}$ in terms of (a) the energy consumption, (b) the throughput, and (c) the energy efficiency, respectively.

The energy efficiency of IEEE 802.11 DCF performs the worst since it always uses the maximum power to transmit, even though it has the best throughput performance. On the contrary, RCRC always uses the minimum transmission power for DATA transmission. Although RCRC has poor throughput when $B$ is approaching to $C$ and $C$ is located in the $I R$ of $B$, RCRC still has the best energy efficiency. As for RTRC and SCRC, the two protocols have the same throughput. When $D_{A B}$ is shorter than $0.39 * T R\left(P_{\max }\right)$, RTRC has better energy efficiency than SCRC since the energy consumption of RTRC is less than that of SCRC. Otherwise, SCRC has better energy efficiency than RTRC. It is worth noting that the energy efficiencies of RTRC and SCRC have the same performance with that of IEEE 802.11 DCF when $D_{A B}$ is respectively larger than 0.56 and 0.72 times the maximum transmission range. It is because, in those situations, RTRC and SCRC will use the maximum power to transmit.

\section{CONCLUSIONS}

The paper analyses the relationships among the transmission range, carrier-sensing range, and interference range under different transmission power level and reveals that the transmission range of the sender, transmission range of the receiver, the carrier-sensing range of the sender, and furthermore the carrier-sensing range of the receiver can be used to cover the interference range in order to avoid the POINT problem. Thus, STRC, RTRC, SCRC, and RCRC MAC protocols are presented and analyzed. An adaptive range-based power control (ARPC) MAC protocol for collision avoidance in wireless ad hoc networks is proposed as well, which makes use of the advantages of the four mechanisms to reduce energy consumption and avoid collisions. Simulation results show that ARPC can not only reduce the energy consumption without sacrificing network throughput, but also avoid the POINT problem accordingly. As a consequence, ARPC is an effective and efficient power control MAC protocol in wireless ad hoc networks.

\section{ACKNOWLEDGEMENT}

This work was partially supported by National Science Council of the Republic of China under Grant NSC 95-2221-
E-032-005 and NSC 95-2524-S-032-003.

\section{REFERENCES}

[1] J. Gomez, A. T. Campbell, M. Naghshineh, and C. Bisdikian, "Conserving transmission power in wireless ad hoc networks," in Proceedings of the IEEE International Conference on Network Protocols (ICNP), 2001, pp. 24-34.

[2] S.-L. Wu, Y.-C. Tseng, C.-Y. Lin, and J.-P. Sheu, "A multi-channel MAC protocol with power control for multi-hop mobile ad hoc networks," Computer Journal, vol. 45, no. 1, pp. 101-110, 2002.

[3] E. S. Jung and N. H. Vaidya, "A power control MAC protocol for ad hoc networks," in Proceedings of the ACM International Conference on Mobile Computing and Networking (MOBICOM), 2002, pp. 36-47.

[4] A. Muqattash and M. Krunz, "A single-channel solution for transmission power control in wireless ad hoc networks," in Proceedings of the ACM International Symposium on Mobile Ad Hoc Networking and Computing (MOBIHOC), 2004, pp. 210-221.

[5] S.-L. Wu, Y.-C. Tseng, and J.-P. Sheu, "Intelligent medium access for mobile ad hoc networks with busy tones and power control," IEEE Journal on Selected Areas in Communications, vol. 18, no. 9, pp. 1647$1657,2000$.

[6] K.-P. Shih and Y.-D. Chen, "CAPC: A collision avoidance power control MAC protocol for wireless ad hoc networks," IEEE Communications Letters, vol. 9, no. 9, pp. 859-861, 2005.

[7] J. Zhu, X. Guo, L. L. Yang, and W. Steven, "Leveraging spatial reuse in 802.11 mesh networks with enhanced physical carrier sensing," in Proceedings of the IEEE International Conference on Communications (ICC), vol. 7, 2004, pp. 4004-4011.

[8] D. Qiao, S. Choi, A. Jain, and K. G. Shin, "Miser: An optimal lowenergy transmission strategy for IEEE $802.11 \mathrm{a} / \mathrm{h}$," in Proceedings of the ACM International Conference on Mobile Computing and Networking (MOBICOM), 2003, pp. 161-175.

[9] J. Zhang, Z. Fang, and B. Brahim, "Adaptive power control for single channel ad hoc networks," in Proceedings of the IEEE International Conference on Communications (ICC), 2005, pp. 3156-3160.

[10] Y. Zhou and S. M. Nettles, "Balancing the hidden and exposed node problems with power control in CSMA/CA-based wireless networks," in Proceedings of the IEEE Wireless Communications and Networking Conference (WCNC), 2005, pp. 683-688.

[11] K. Xu, M. Gerla, and S. Bae, "How effective is the IEEE 802.11 RTS/CTS handshake in ad hoc networks?" in Proceedings of the IEEE Global Telecommunications Conference (GLOBECOM), vol. 1, 2002, pp. 72-76.

[12] T. Rappaport, Wireless Communications: Principles and Practice (2nd Edition). Prentice Hall, New Jersey, 2002.

[13] IEEE Std 802.11-1999, Part 11: Wireless LAN Medium Access Control (MAC) and Physical Layer (PHY) Specifications, IEEE, Aug. 1999.

[14] The Network Simulator - 2, [Online] Available: http://www.isi.edu/nsnam/ns/.

[15] J. Shi, T. Salonidis, and E. W. Knightly, "Starvation mitigation through multi-channel coordination in CSMA multi-hop wireless networks," in Proceedings of the ACM International Symposium on Mobile Ad Hoc Networking and Computing (MOBIHOC), 2006. 